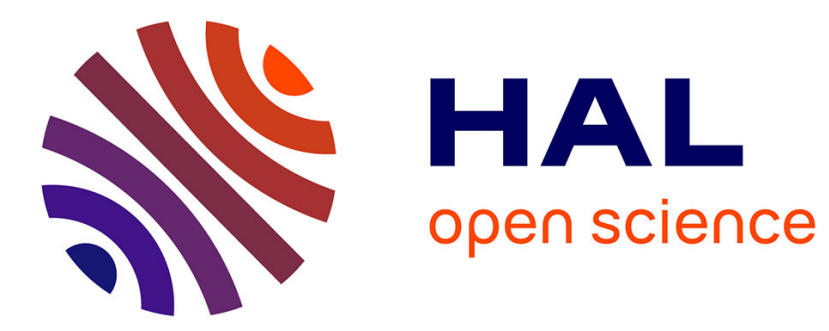

\title{
3D genome organisation in Drosophila
}

Charlotte Moretti, Isabelle Stévant, Yad Ghavi-Helm

\section{To cite this version:}

Charlotte Moretti, Isabelle Stévant, Yad Ghavi-Helm. 3D genome organisation in Drosophila. Briefings in Functional Genomics and Proteomics, 2019, 10.1093/bfgp/elz029 . hal-02408007

\section{HAL Id: hal-02408007 https://hal.science/hal-02408007}

Submitted on 17 Dec 2019

HAL is a multi-disciplinary open access archive for the deposit and dissemination of scientific research documents, whether they are published or not. The documents may come from teaching and research institutions in France or abroad, or from public or private research centers.
L'archive ouverte pluridisciplinaire HAL, est destinée au dépôt et à la diffusion de documents scientifiques de niveau recherche, publiés ou non, émanant des établissements d'enseignement et de recherche français ou étrangers, des laboratoires publics ou privés. 
3D genome organisation in Drosophila

2

3

4

5

Charlotte Moretti†, Isabelle Stévant ${ }^{\dagger}$, Yad Ghavi-Helm*

6

7

8

9 Affiliation

10 Institut de Génomique Fonctionnelle de Lyon, Univ Lyon, CNRS UMR 5242, Ecole Normale Supérieure de

11 Lyon, Université Claude Bernard Lyon 1, 46 allée d'Italie F-69364 Lyon, France

12

13 TThese authors contributed equally

14

$15 *$ Contact information

16 Email yad.ghavi-helm@ens-lyon.fr

17 Telephone $\quad+33426731350$ 
19 Ever since Thomas Hunt Morgan's discovery of the chromosomal basis of inheritance by using Drosophila 20 melanogaster as a model organism, the fruit fly has remained an essential model system in studies of 21 genome biology, including chromatin organisation. Very much as in vertebrates, in Drosophila the 22 genome is organised in territories, compartments, and topologically-associated domains (TADs). 23 However, these domains might be formed through a slightly different mechanism than in vertebrates 24 due to the presence of a large and potentially redundant set of insulator proteins and the minor role of 25 dCTCF in TAD boundary formation. Here, we review the different levels of chromatin organisation in Drosophila and discuss mechanisms and factors that might be involved in TAD formation. The dynamics of TADs and enhancer-promoter interactions in the context of transcription are covered in the light of currently conflicting results. Finally, we illustrate the value of polymer modelling approaches to infer the principles governing the 3-dimensional organisation of the Drosophila genome.

\section{Authors biography}

32 Charlotte Moretti is a postdoctoral fellow in the Ghavi-Helm team. She received her Ph.D. in biology 33 from the René Descartes University in Paris.

34 Isabelle Stévant is a postdoctoral fellow in the Ghavi-Helm team. She received her Ph.D. in 35 bioinformatics from the University of Geneva.

36 Yad Ghavi-Helm is a group leader of the Centre National de la Recherche Scientifique (CNRS) at the 37 Institute of Functional Genomics of Lyon (IGFL). Her team focuses on transcription regulation, chromatin 38 organisation, and enhancer-promoter interaction functionality during early Drosophila embryogenesis.

\section{Key points}

41 - Drosophila is a key model system to study the 3-dimensional organisation of the genome

42 - The Drosophila genome is organised in territories, A/B compartments, and topologically-associated domains (TADs)

44 - The relative contribution of transcription, architectural proteins, and epigenetics domains in TAD formation remains unclear

46 - Some TAD borders and chromatin loops appear dynamic across conditions, while others are stable 
49 Keywords

50 Chromatin organisation; Drosophila; topologically-associated domains; transcription 
52 The tight regulation of gene expression during development, cell differentiation, and cell identity 53 maintenance involves the 3-dimensional (3D) organisation of the genome. This organisation is apparent 54 at different levels, from large chromosome territories and compartments to more restricted structures 55 such as TADs and local loops (Figure 1). As general principles of chromatin organisation are largely conserved across metazoans, Drosophila melanogaster rapidly emerged as a model of choice for the

57 field. Over the years, fly researchers have developed an extremely flexible genetic toolbox including 58 balancer chromosomes, the GAL4/UAS expression system, efficient transgenesis, and genome editing by CRISPR-Cas9 [1]. In addition, the Drosophila genome is relatively small (180 Mb with only three pairs of autosomes) and thoroughly annotated (it was one of the first genomes to be sequenced, in 2000 [2]).

61 Overall, this makes Drosophila an excellent model organism to study chromatin organisation. In this review, we first introduce the different levels of chromatin organisation in Drosophila nuclei, before describing in detail TAD borders and their dynamics with respect to transcription. We conclude by illustrating the benefits of polymer modelling in deciphering the essential mechanisms underlying TAD formation in Drosophila.

66

\section{Different levels of chromatin organisation}

68 During interphase, eukaryotic chromosomes are confined within the nucleus, where a globally compact and efficient folding of chromatin is enforced, while appropriate regions remain accessible for normal gene expression. This compaction clearly does not occur at random. Since the $19^{\text {th }}$ century, thanks to the rapid development of microscopy, we know that chromosomes occupy different territories within the nucleus (Figure 1a, reviewed in [3]). They are locally constrained [4] and segregated in different nuclear regions, with partial and stochastic overlaps in the periphery of the territories [5]. These territories are not identical from cell to cell, nonetheless large and gene-poor chromosomes tend to be located close to the periphery of the nucleus, while short and gene-rich chromosomes are more frequently found at the centre of the nucleus. Similarly, the level of folding and compaction of chromosomes depends on their localisation within the nucleus. At the periphery of the nucleus, chromatin is associated to the nuclear lamina and appears very compact and generally transcriptionally inactive, while closer to the centre of the nucleus chromatin is less compact and enriched in transcriptionally active regions [6-8]. Clusters of genes associated with the nuclear lamina are dynamically co-regulated during development via a

81 transient reduction of Lamin binding and relocation towards the nuclear interior [6]. In addition, 82 Drosophila chromosomes adopt a polarised orientation, known as the Rabl configuration, with 
centromeres and telomeres clustering separately at two opposite nuclear poles (Figure 1a) [4,5,9-11]. Finally, in Drosophila genomes, homologous chromosomes are paired throughout the cell cycle, interacting with each other in tight and loose trans-interactions $[12,13]$. As a consequence, gene expression can be affected by a phenomenon called transvection [14], where cis-regulatory elements regulate the expression of genes located on the homologous chromosome [15].

The recent development of the chromosome conformation capture (3C) technique and its genome-wide derivatives (such as $\mathrm{Hi}-\mathrm{C}$ ) allows the identification, in a high-throughput manner, of genomic regions that are in close proximity in vivo. Seminal Hi-C experiments performed in Drosophila embryos revealed the presence of large (100 kb on average) and discrete domains along the linear chromosomes [16]. Such "topologically-associated domains" (TADs), are defined as chromosomal regional displaying a high selfinteraction frequency delimited by a dramatic decrease of interaction frequencies with the regions on either side of the domains that defines their boundaries (Figure $\mathbf{1 b}, \mathbf{c}$ ). $\mathrm{Hi}-\mathrm{C}$ experiments also revealed a segregation of the genome into two types of compartments, called A and B compartments, which represent the first principal component of the $\mathrm{Hi}-\mathrm{C}$ contact matrix and can be visualised as a plaid-like pattern on Hi-C contact maps (Figure 1b) [17]. The A compartment contains gene-rich and transcriptionally active open chromatin, while the B compartment is gene-poor and enriched for closed chromatin regions [17]. Compartments tend to interact more frequently within their own class, probably owing to the preferential affinity of the protein set carried on each compartment for their siblings.

TADs and compartments can be classified into four main classes according to their global chromatin signature: one active class (corresponding to the $A$ compartment) and three inactive classes (corresponding to the B compartment). Indeed, TADs strongly correlate with epigenetic domains, and their boundaries are enriched with active histone post-translational modifications and insulator protein binding sites $[16,18]$ (developed below). While chromatin state is quite consistent within a same TAD, the segregation of active and repressive marks is not always perfect and TADs can display heterogeneous chromatin states [19]. Active TADs are enriched in transcriptionally active genes and therefore display active chromatin marks (H3K4me3 and H3K36me3) [20]. Inactive TADs are more frequently found associated with the nuclear lamina compared to active TADs [6]. The first class of inactive TADs corresponds to Polycomb-repressed loci that are enriched in histone $\mathrm{H} 3$ trimethylated at lysine 27 (H3K27me3) $[16,18,21]$. These Polycomb-associated TADs represent approximately $10 \%$ of the fly genome and contain a significant number of developmental genes and transcription factors, notably involved in tissue patterning. The second class of inactive TADs is composed of heterochromatin enriched in H3K9me2, HP1, and Su(var)3-9 [16]. Finally, the third class, covering up to $50 \%$ of the 
115 euchromatin, is devoid of specifically-enriched chromatin marks $[16,20,22]$. However, recent studies 116 showed that the $\mathrm{H} 3 \mathrm{~K} 27 \mathrm{me} 2$ chromatin mark is pervasive in transcriptionally repressed regions and thus 117 is likely to be present in this "void" inactive TAD class $[23,24]$.

118 TADs and compartments are evolutionarily conserved structures [25] present in a large variety of 119 species, including mammals [26,27], zebrafish [28], Caenorhabditis elegans [29], and yeast [30,31], 120 suggesting that they represent robust functional units of the genome. Drosophila TADs are generally 121 smaller than mammalian TADs (1 Mb on average in mouse and human). However, the detection of TADs 122 is highly dependent on the resolution of the Hi-C assays and the detection threshold used in the TAD 123 discovery algorithms, generating a large discrepancy between studies. Indeed, first reports indicated that 124 the Drosophila genome may contain about 1300 TADs $[16,18]$, while more recent studies using higher 125 resolution $\mathrm{Hi}-\mathrm{C}$ assays distinguished 2000 to 4000 or more TADs [32-34]. Because TADs are weakly 126 defined at many loci, it has been proposed that the Drosophila genome is partitioned in TADs that are 127 separated either by short boundaries or by longer unstructured "inter-TADs" regions [19]. More recently, 128 it was reported that these inter-TAD regions actually contain small contiguous active TADs with an 129 average size of tens of kilobases [34], which might establish gene loops between transcriptional start and 130 termination sites, and have also been referred to as "mini-domains" [17]. Larger TADs would instead be 131 enriched in inactive regions [32]. In addition, TADs and inter-TADs have been shown to correspond to the 132 cytological bands and interbands of the polytene chromosomes [19,35-37].

133 In addition to the uncertainty in the number and location of TAD boundaries, their actual function in 134 transcription regulation is also heavily debated. TADs are thought to define isolated/insulated structural 135 domains within which chromatin looping brings genes in close proximity with their regulators. Indeed, 136 the expression of genes located within the same TAD is more highly correlated than that of genes 137 separated by TAD boundaries [32], probably because cis-regulatory elements are predominantly located 138 in the same TADs as their target promoters. However, engineered chromosomal inversions affecting the 139 genomic organisation of three testis-specific gene clusters or deficiencies affecting the left arm of 140 chromosome 2 only mildly affect gene expression [38,39]. Similarly, the analysis of nested chromosomal 141 inversions present within the second and third chromosomes of a highly rearranged "balancer" line 142 revealed that changes in chromatin organisation and changes in gene expression are largely uncoupled 143 [40]. At many loci, breaking, shuffling, or fusing TADs can have surprisingly little effect on gene 144 expression, suggesting that TAD boundaries are not always essential for correct gene expression [40]. 145 The role of chromatin organisation in the regulation of gene expression appears to be more complex 146 than previously thought. 
147 Within TADs, chromatin loops between regulatory elements are involved in the fine regulation of gene 148 expression. Genes are positively regulated through enhancer-promoter interactions, where enhancers 149 are brought in close proximity to their target promoter(s) and activate transcription via the release of 150 paused RNA polymerase II (RNA Pol II) [41] (Figure 1d). In some cases, distal enhancer-promoter 151 interactions are facilitated by the pairing of insulator proteins that dimerise to form a loop $[42,43]$. In 152 other cases, enhancer-promoter interactions have been associated with the presence of paused RNA Pol

153 II [41]. While most chromatin interactions are limited within a single $T A D$, examples of long-range 154 contacts across TAD boundaries have also been reported [16]. Some of these long-range contacts involve 155 Polycomb domains, initially visualised by microscopy as distinct foci called "Polycomb bodies". Long156 range interactions driven by Polycomb-mediated looping are responsible for the negative regulation of 157 developmental gene expression. These interactions involve Polycomb response elements (PREs) and the 158 recruitment of the Polycomb repressive complex PRC1 [33,44-48] (extensively reviewed in [49]). Long159 range gene-gene interactions can also involve non-Polycomb repressed genes [36], some of which can be 160 co-expressed, such as the hbs (hibris) and sns (sticks and stones) genes [16,40].

\section{Drivers of chromatin organisation}

163 The genetic and epigenetic rules that may drive TAD formation and maintenance have been the subject

164 of intense research in recent years. In particular, great efforts have been directed towards the precise 165 identification and description of TAD boundaries and the different factors bound in those regions, to 166 disclose the molecular mechanisms at stake.

\section{Active chromatin and transcription}

168 While TADs can be classified based on their respective epigenetic states, their borders exhibit constant 169 properties: they tend to be located in gene-rich and highly-transcribed open chromatin regions 170 characterised by the presence of particular chromatin marks. More specifically, TAD boundaries are 171 enriched with active chromatin marks such as H3K27ac, H3K4me1, H3K4me3, H3K36me3, and H4K16ac $172[16,18,19,32,50]$, and depleted in the repressive H3K27me3 chromatin mark [24,36]. Boundaries are also 173 particularly sensitive to DNase I treatment, a hallmark of open chromatin, and thus significantly depleted 174 in nucleosomes [36]. In addition, the RNA Pol II itself $[19,51]$ as well as the general transcription factor 175 TFIIC [36] are enriched at TAD boundaries. snoRNAs, known to mediate the stable opening of chromatin 176 [52], have also been identified as positive drivers for boundary demarcation [53]. A vast majority (77\%) 177 of boundaries overlap with the promoter of ubiquitous, stable, and highly-expressed housekeeping 178 genes and their enhancers $[16,18,19,32,51,54]$. Tissue-specific enhancers are also occasionally associated 
179 with TAD borders [19,54]. Conversely, tightly-regulated developmental genes are predominantly 180 embedded in H3K27me3-rich domains, which mostly correspond to inactive Polycomb-repressed 181 domains [24]. This enrichment of actively transcribed genes at TAD borders suggests that transcription 182 per se might be involved in their formation. In line with this hypothesis, simulated Hi-C maps based on 183 GRO-seq data can accurately recapitulate contact heat maps [17]. Besides, the genome-wide inhibition 184 of transcription observed in cells exposed to a temperature stress (heat shock) is accompanied by a 185 drastic reorganisation of TAD borders and a general decrease in border strength [55]. However, 186 transcription might not be the sole driver of TAD boundary formation, as suggested by the relative 187 absence of coordinated changes in TAD boundary formation upon chemical perturbation of gene 188 expression. Indeed, a more direct transcriptional inhibition with flavopiridol or triptolide causes a milder 189 decrease in border strength $[17,55]$, and inhibiting transcription by triptolide does not prevent the 190 establishment of chromatin organisation during genome activation in early embryos [51]. Furthermore, 191 the consistency of TAD border coordinates across different sections of stage 5 embryos, suggests an 192 uncoupling between boundary formation and transcriptional programs [36], and calls for other 193 mechanisms than transcription to be involved in TAD boundary formation. Actually, the deletion of two 194 different 3 and 4 kb-long regions, each encompassing a different TAD border bound by multiple insulator 195 proteins (including dCTCF and CP190) as well as the Rad21 cohesin subunit, was sufficient to cause TAD 196 fusion [56]. It has thus been proposed that architectural proteins such as insulators might be better 197 predictors of TAD borders than transcription itself [34,55].

198 Insulators and other architectural proteins

199 Insulators were initially identified through their ability to prevent long-range interactions between 200 promoters and enhancers when positioned between them [57-60], and to block the spreading of 201 repressive epigenetic marks [61-65]. More recently, $\mathrm{Hi}-\mathrm{C}$ experiments performed on Drosophila embryos 202 [16] and Kc167cells [18], revealed that some insulator proteins are significantly enriched at TAD borders, 203 suggesting a more global role in chromatin organisation.

204 Multiple studies have confirmed that insulators and other architectural proteins, including the B box 205 binding subunit of TFIIIC, are enriched at TAD borders [19,32,34,36,53-55,66]. Whereas vertebrate 206 genomes are characterised by the preponderant role of the zinc finger CTCF insulator protein [67], in 207 Drosophila, a large number of insulator proteins have been identified, including dCTCF, BEAF-32, CP190, 208 Chromator, ZW5, mod(mdg4), GAF, M1BP, Su(Hw), Ibf1, Ibf2, Pita, ZIPIC etc. [68]. These insulator 209 proteins can be divided in different classes, each binding different regions of the genome [69]. A large 210 number of proteins are referred to as "insulators" in Drosophila, although their actual insulation function 
211 has often not been clearly established. Many insulators might in fact be involved in transcription

212 regulation rather than in chromatin organisation and TAD boundary formation [70]. Proteins involved in

213 chromatin organisation are thus globally referred to as "architectural proteins".

214 dCTCF, the main driver of TAD formation in vertebrates, is enriched at gene promoters, 5' UTRs, and

215 intergenic regions, suggesting that it might be involved in the regulation of gene expression [69]. Yet, it is

216 only found at $28 \%$ of TAD borders, with no evidence for a specific motif orientation contrary to what is

217 observed in vertebrates [17]. Moreover, it is not required during embryonic development, and its

218 deletion is not lethal until late pupal stages. Instead, it is involved in the correct expression of Hox genes,

219 during both embryogenesis and larvae stages [71]. The relatively minor role of dCTCF in Drosophila is

220 surprising given the high enrichment of CTCF at TAD borders in vertebrates and its proposed role, in

221 combination with the cohesin complex, in TAD formation through the loop extrusion mechanism [67]. In

222 Drosophila, the cohesin complex is enriched at TAD boundaries, as expected from its location at the

223 promoter of active genes [66,72], and could therefore also participate in TAD formation.. However, a

224 concerted action between dCTCF and cohesin in Drosophila remains uncertain. Indeed, initial

225 experiments suggested that only $25 \%$ of dCTCF binding sites overlap with the binding of the Stromalin

226 cohesin subunit [72], while a latter study found that nearly 50\% of dCTCF binding sites overlap with the

227 binding of the Rad21 cohesin subunit [66]. In addition to its potential role in TAD formation, cohesin

228 might be involved in long-range enhancer-promoter interactions. Indeed, the cohesin complex, and its

229 loading factor Nipped-B, are bound to the promoter of many active [73] and paused [74] genes, as well

230 as to enhancers $[66,75]$. Moreover, Nipped-B was identified in a genetic screen designed to detect

231 architectural proteins involved in the activation of the cut gene by its remote wing enhancer [76].

232 Cohesin has also been proposed to mediate interactions between Polycomb response elements (PREs)

233 by controlling the binding of the Polycomb Repressive Complex 1 (PCR1) [77]. Altogether, while cohesin

234 probably has an important role in regulating gene expression, for example by promoting the formation

235 of mini-domains [78] or by mediating long-range enhancer-promoter interactions, its role in TAD

236 formation through loop extrusion has not been demonstrated to date. In addition, condensin II, which

237 largely co-localizes with cohesin [66], might act as an antagonist to cohesin [78].

238 In flies, other architectural proteins such as BEAF-32, CP190, and Chromator are probably more 239 important than dCTCF in TAD boundary formation. In particular, CP190 and BEAF-32, enriched at gene 240 promoters, $5^{\prime}$ UTRs, and intergenic regions [69], are likely involved in TAD boundary formation. In 241 synchronised cells, approximately $75 \%$ of the protein pairs BEAF-32/Chromator and BEAF-32/CP190 are 242 found at TAD borders [34]. Conversely, 77\% of TAD borders harbour these two protein pairs [34]. 
243 Additionally, expressing the dominant-negative BEAF-32 protein in the salivary glands resulted in less 244 condensed and 'puffy' polytene chromosomes [79]. However, siRNA depletion of BEAF-32 in S2 cells has 245 no significant effect on TAD boundaries and chromatin interactions [32], a discrepancy that could be 246 explained by the redundancy between BEAF-32 and the transcription factor DREF, which are sharing the 247 exact same binding sites and might play redundant roles in chromatin organisation at TAD borders [32].

248 A similar 'puffy' polytene chromosome phenotype was also observed upon depletion of the JIL-1 histone 249 H3S10 kinase [80]. Interestingly, Chromator, which recruits JIL-1 on polytene chromosomes [81], is found 250 significantly enriched at TAD borders $[34,50]$. Altogether, these results suggest that many factors could 251 act as architectural proteins in TAD border formation. This idea is supported by in silico enhancer252 blocking assays, that estimate the blocking effect of combinations of different proteins and DNA-binding 253 motifs [82]. It is likely that TAD border strength, defined as the ratio of intra-versus inter-TAD interaction 254 frequencies, correlates directly with the number and combination of architectural proteins bound at TAD 255 borders $[32,34,53,55,66,82]$. In this view, architectural proteins would act synergistically, creating 256 regulatory hubs of highly- or lowly- occupied borders that correspond to more stable or more dynamic 257 TADs.

\section{Dynamics of chromatin organisation}

260 After fertilisation, early Drosophila embryos develop as a syncytium through 13 rapid and synchronised 261 nuclear divisions (in about 120 minutes), before cellularisation occurs at nuclear cycle (nc) 14 [83]. While 262 zygotic transcription already starts at nc8, the major wave of zygotic transcription occurs at nc14. The 263 Drosophila genome becomes gradually compartmentalised from nc12 (about 100 minutes after egg lay) 264 into TADs, a process that continues throughout zygotic genome activation (ZGA) $[44,51,84]$, despite 265 being interspersed by TAD disruptions at each cell cycle (Figure 2). Although not well separated until late 266 embryogenesis, active and Polycomb-repressed compartments start to appear soon after the major 267 wave of ZGA, reflecting the dynamic establishment of chromatin organisation during embryogenesis 268 [44]. Importantly, RNA Pol II is enriched at the boundary of newly-formed TADs and the dynamics of its 269 recruitment strongly correlates with TAD formation. However, chemical inhibition of transcription with 270 alpha-amanitin or triptolide before the onset of zygotic transcription (before nc8) showed that TADs can 271 form independently of transcription, albeit with changes in intra- and inter-TAD organisation [51]. At 272 nc12, in addition to RNA Pol II, TAD borders are also enriched for the binding of Zelda, a pioneering 273 transcription factor essential to open chromatin and activate early gene expression during ZGA $[85,86]$. 274 However, the depletion of Zelda only results in a partial loss of TAD boundaries at Zelda-specific TAD 
275 boundaries, demonstrating that its possible role in TAD boundary formation is limited to only a subset of 276 loci. Zelda is also required to induce long-range active gene contacts during the midblastula transition 277 (nc9 to nc13). Soon afterwards it associates with GAF to determine the open chromatin structures of 278 active gene promoters and insulators [44,86]. Starting from nc14, GAF also plays a role in forming 279 repressive short-distance loops within Polycomb domains, potentially via the recruitment of PRC1 280 proteins [44].

281 During cell type specification, it has been postulated that TADs might be partially rearranged in order to 282 promote the expression of cell-type specific genes and repress alternative cell fate choices. Comparing 283 Hi-C data from Kc167 cells (a 8-12h embryo-derived cell line) to BG3 differentiated neuronal cells, 284 revealed that only some TADs are conserved in both cell lines, while others are cell-type specific [87]. 285 Besides, enhancers controlled by neuronal-specific transcription factors are enriched in neuronal-specific 286 TADs, suggesting that this change in topology is driven by cell differentiation [87]. However, comparing $287 \mathrm{Hi}-\mathrm{C}$ data from Kc167 cells to either fly embryos or polytene chromosomes (from salivary glands of third 288 instar larvae) revealed that TADs are invariant across these different conditions [18,37]. These 289 apparently conflicting results might be explained by discrepancies in the identification of TADs between $290 \mathrm{Hi}-\mathrm{C}$ methods and datasets sequenced at different depths, as explained above.

291 Microscopy-based assays offer an interesting alternative to compare chromatin organisation across 292 conditions and assess the timing of TAD formation with respect to transcription in single cells. Such an 293 approach (Hi-M) in nc14 Drosophila embryos revealed important intra-TAD rearrangements at the snail 294 locus when comparing snail-expressing and non-expressing cells [84]. Intra-TAD interactions, such as 295 enhancer-promoter interactions, could therefore be much more dynamic and correlated to transcription 296 than TADs boundaries. However, comparing enhancer-promoter interactions at two early embryogenesis 297 stages (3-4h and 6-8h after egg lay) uncovered much less dynamics than expected from transcriptional 298 changes [41]. Unexpectedly, enhancers are already in close proximity to the promoter of their target 299 gene prior to gene expression and these stable interactions are associated with the presence of paused 300 RNA Pol II at the promoter. This suggests that enhancer-promoter interactions can be pre-formed before 301 gene expression, and that these paused transcriptional hubs can be rapidly activated upon the 302 recruitment of additional co-factors to release the paused polymerase [41]. Stable interactions might 303 however be specific to early stages of embryogenesis, characterised by rapid cell cycles and extensive 304 RNA Pol II pausing at gene promoters. In addition, comparing sorted mesodermal nuclei to whole 305 embryos revealed that enhancer-promoter interactions may form in tissues where the gene will never be 306 expressed [41]. The artificial tethering of eve enhancers to a reporter gene using homie insulator 
307 elements confirmed that the close proximity between an enhancer and its target promoter is not 308 sufficient to activate transcription [43]. However, physical proximity is necessary for sustained 309 transcription, which in turn stabilizes and reinforces the physical association of paired regulatory 310 elements [43]. Besides, this physical association might not necessarily imply direct contact between a 311 distal enhancer and its promoter target(s). Indeed, a recent study based on live-cell imaging reported 312 that co-expressed genes sharing the same enhancer are separated by large distances (at least 100-200 $313 \mathrm{~nm}$ ) during transcription [88]. The recruitment of numerous transcription factors and associated co-

314 factors that regulate gene expression could prevent any direct contact [88].

\section{Using polymer physics to model chromatin organisation}

317 The association of recent high-resolution techniques, namely $\mathrm{Hi}-\mathrm{C}[16,18,34]$ and super-resolution 318 imaging [89], unambiguously demonstrated a strong link between the 1D chromatin epigenetic 319 landscape and its 3D conformation inside the nucleus. However, it is still unclear whether chromatin 320 organisation is a by-product of epigenetic marks and transcription or is genuinely involved in regulating 321 gene expression. Computational modelling proposes an attractive approach to investigate this question 322 and infer which factors are essential to chromatin organisation.

323 Modelling treats chromatin as a semi-flexible, self-avoiding, and self-interacting copolymer obeying basic 324 constraints of thermal motion and steric repulsion. This copolymer is composed of blocks of consecutive 325 monomers sharing the same epigenetic state. Each monomer (i.e. genomic region) preferentially 326 interacts with other segments in the same epigenetic state. Applying polymer modelling to Drosophila 327 chromosomes remarkably recapitulates the organisation of the fly genome into TADs, as well as A and B 328 compartmental organisation [31,80-82]. In this model, transcriptionally inactive 'null' domains, devoid of 329 specific epigenetic marks, behave as polymers in a fractal globule [89]. There, nucleosome-nucleosome 330 interactions are thought to be a dominant, potentially sufficient, mechanism for the high compaction of 331 inactive domains [92]. Conversely, the relatively decondensed active domains might correspond to low 332 nucleosome interactions $[19,89,92,93]$. Experimentally, the acetylation of histones is known to promote 333 chromatin unfolding by decreasing nucleosome-nucleosome interactions, as the neutralisation of 334 positive charges on histone tails on a nucleosome hinders interaction with the acidic patch of another 335 nucleosome [94]. Besides nucleosome-nucleosome interactions, computational analyses give strong 336 interaction values for Polycomb-repressed domains, suggesting a prominent role for the molecular 337 bridging action of Polycomb-group proteins and linker histone $\mathrm{H} 1[89,92]$. 
338 Although epigenetic modifications appear to be primary drivers of spatial organisation, recent studies

339 suggest that precise spatial organisation strongly influences the stability and spreading of epigenetic

340 states [91,95-97]. Chromatin folding would create hubs enriched for specific regulatory proteins,

341 increasing the local concentration of these molecular actors and their binding affinities. These "bio-

342 chemical nano-reactors" would participate in the trans spreading of an epigenetic state to other

343 chromatin segments [96]. Importantly, current models assuming that chromatin organisation is solely

344 driven by the epigenetic state of interacting domains are certainly over-simplified. Indeed, the presence

345 of TAD borders between segments in the same epigenetic state demonstrates that TAD formation

346 simulations should include additional mechanisms besides homotypic chromatin folding [56].

347 Nevertheless, polymer modelling is a valuable tool to challenge the current biological hypotheses

348 regarding TADs formation during embryonic development, differentiation, and cell cycle. In addition, the

349 theoretical framework developed in computational modelling studies will enable investigations under

350 perturbed conditions and undoubtedly provide clues regarding the identity and function of essential

351 factors driving chromatin organisation.

\section{Concluding remarks}

354 Over the last few years, sustained efforts have been carried out to precisely describe and understand the 355 mechanisms driving TAD formation and dynamics. Complementary approaches, such as $\mathrm{Hi}-\mathrm{C}$ 356 experiments, super-resolution imaging, and in silico modelling have allowed an integrative and powerful 357 analysis of spatial chromatin organisation in various model organisms, from yeast to human. In 358 Drosophila, TAD formation has been proposed to arise from the combinatorial action of (i) specific 359 interactions between chromatin segments sharing the same epigenetic state, (ii) binding of insulators, 360 chromatin remodelers, and other architectural proteins at TAD orders, and (iii) active transcription. This 361 combination of mechanisms could explain the robustness of TAD organisation and the mild phenotypes 362 observed upon challenges as diverse as protein depletion, genomic rearrangements, or chemical 363 inhibition. The comparative analysis of TAD organisation in different model organisms also raises a 364 number of puzzling questions. For example, how do insulators and cohesin function in TAD border 365 formation and long-range interactions in Drosophila compared to vertebrates? Is the function of TADs 366 evolutionary conserved? While topological domains are conserved among most metazoan, the 367 mechanisms that organise and maintain them have evolved. Chromatin organisation thus appears to be 368 an important functional feature of the genome rather than a by-product of gene expression and 369 regulation. With a large and expanding toolbox including the maternal knock-out of genes during 
370 embryogenesis, CRISPR-Cas9 induced mutations, super-resolution imaging, and single-cell -omic 371 technologies, Drosophila will certainly remain a model of choice to address outstanding questions 372 regarding the 3D genome organisation.

373

\section{Acknowledgments}

375 We thank Cédric Vaillant and all the members of the Ghavi-Helm laboratory for useful comments. This 376 work was financially supported by an ERC starting grant (759708) and the Ecole Normale Supérieure de 377 Lyon to Y.G.-H., and a Fondation ARC pour la recherche sur le cancer fellowship (PDF20171206672) to 378 C.M. 
380 1. Hales KG, Korey CA, Larracuente AM et al. Genetics on the Fly: A Primer on the Drosophila 381 Model System. Genetics 2015;201:815-42.

382 2. Adams MD, Celniker SE, Holt RA et al. The Genome Sequence of Drosophila melanogaster. 383 Science 2000;287:2185-95.

384 3. Cremer T, Cremer M. Chromosome Territories. Cold Spring Harb Perspect Biol $385 \quad 2010 ; 2: a 003889$.

4. Marshall WF, Straight A, Marko JF et al. Interphase chromosomes undergo constrained diffusional motion in living cells. Curr Biol CB 1997;7:930-9.

5. Hochstrasser M, Mathog D, Gruenbaum Y et al. Spatial organization of chromosomes in the salivary gland nuclei of Drosophila melanogaster. J Cell Biol 1986;102:112-23.

390 6. Pickersgill H, Kalverda B, Wit E de et al. Characterization of the Drosophila melanogaster 391 genome at the nuclear lamina. Nat Genet 2006;38:1005.

7. Marshall WF, Dernburg AF, Harmon B et al. Specific interactions of chromatin with the nuclear envelope: positional determination within the nucleus in Drosophila melanogaster. $\mathrm{Mol}$ Biol Cell 1996;7:825-42.

395 8. Boutanaev AM, Mikhaylova LM, Nurminsky DI. The Pattern of Chromosome Folding in 396 Interphase Is Outlined by the Linear Gene Density Profile. Mol Cell Biol 2005;25:8379-86.

397 9. Padeken J, Mendiburo MJ, Chlamydas S et al. The Nucleoplasmin Homolog NLP Mediates 398 Centromere Clustering and Anchoring to the Nucleolus. Mol Cell 2013;50:236-49.

399 10. Lowenstein MG, Goddard TD, Sedat JW. Long-Range Interphase Chromosome Organization 400 in Drosophila: A Study Using Color Barcoded Fluorescence In Situ Hybridization and Structural 401 Clustering Analysis. Mol Biol Cell 2004;15:5678-92.

402 11. Li Q, Tjong $\mathrm{H}, \mathrm{Li} \mathrm{X}$ et al. The three-dimensional genome organization of Drosophila 403

404 12. AlHaj Abed J, Erceg J, Goloborodko A et al. Highly Structured Homolog Pairing Reflects 405 Functional Organization of the Drosophila Genome. Genetics, 2018.

406 13. Erceg J, AlHaj Abed J, Goloborodko A et al. The Genome-Wide, Multi-Layered Architecture 407 of Chromosome Pairing in Early Drosophila Embryos. Genetics, 2018.

408 14. Duncan IW. Transvection effects in Drosophila. Annu Rev Genet 2002;36:521-56.

409 15. Lim B, Heist T, Levine M et al. Visualization of Transvection in Living Drosophila Embryos. 410 Mol Cell 2018;70:287-296.e6. 
411 16. Sexton $\mathrm{T}$, Yaffe E, Kenigsberg E et al. Three-Dimensional Folding and Functional 412 Organization Principles of the Drosophila Genome. Cell 2012;148:458-72.

413 17. Rowley MJ, Nichols MH, Lyu X et al. Evolutionarily Conserved Principles Predict 3D 414 Chromatin Organization. Mol Cell 2017;67:837-852.e7.

415 18. Hou C, Li L, Qin ZS et al. Gene Density, Transcription, and Insulators Contribute to the 416 Partition of the Drosophila Genome into Physical Domains. Mol Cell 2012;48:471-84.

417 19. Ulianov SV, Khrameeva EE, Gavrilov AA et al. Active chromatin and transcription play a 418 key role in chromosome partitioning into topologically associating domains. Genome Res $419 \quad 2016 ; 26: 70-84$.

420 20. Ho JWK, Jung YL, Liu T et al. Comparative analysis of metazoan chromatin organization. 421 Nature 2014;512:449-52.

422 21. Cheutin T, Cavalli G. Polycomb silencing: from linear chromatin domains to 3D chromosome 423 folding. Curr Opin Genet Dev 2014;25:30-7.

424 22. Filion GJ, van Bemmel JG, Braunschweig U et al. Systematic protein location mapping 425 reveals five principal chromatin types in Drosophila cells. Cell 2010;143:212-24.

426 23. Lee H-G, Kahn TG, Simcox A et al. Genome-wide activities of Polycomb complexes control 427 pervasive transcription. Genome Res 2015;25:1170-81.

428 24. El-Sharnouby S, Fischer B, Magbanua JP et al. Regions of very low H3K27me3 partition the 429 Drosophila genome into topological domains. Cuddapah S (ed.). PLOS ONE 2017;12:e0172725.

430 25. Krefting J, Andrade-Navarro MA, Ibn-Salem J. Evolutionary stability of topologically 431 associating domains is associated with conserved gene regulation. BMC Biol 2018;16:87.

432 26. Dixon JR, Selvaraj S, Yue F et al. Topological domains in mammalian genomes identified by 433 analysis of chromatin interactions. Nature 2012;485:376-80.

434 27. Nora EP, Lajoie BR, Schulz EG et al. Spatial partitioning of the regulatory landscape of the 435 X-inactivation centre. Nature 2012;485:381-385.

436 28. Gómez-Marín C, Tena JJ, Acemel RD et al. Evolutionary comparison reveals that diverging 437 CTCF sites are signatures of ancestral topological associating domains borders. Proc Natl Acad 438 Sci 2015;112:7542-7.

439 29. Crane E, Bian Q, McCord RP et al. Condensin-driven remodelling of X chromosome 440 topology during dosage compensation. Nature 2015;523:240-4.

441 30. Mizuguchi T, Fudenberg G, Mehta S et al. Cohesin-dependent globules and heterochromatin 442 shape 3D genome architecture in S. pombe. Nature 2014;516:432-5.

443 31. Hsieh T-HS, Weiner A, Lajoie B et al. Mapping Nucleosome Resolution Chromosome 444 Folding in Yeast by Micro-C. Cell 2015;162:108-19. 

underlying genome organization in flies. Nat Commun 2018;9:189.

447 33. Eagen KP, Aiden EL, Kornberg RD. Polycomb-mediated chromatin loops revealed by a subkilobase-resolution chromatin interaction map. Proc Natl Acad Sci 2017:201701291.

34. Wang Q, Sun Q, Czajkowsky DM et al. Sub-kb Hi-C in D . melanogaster reveals conserved characteristics of TADs between insect and mammalian cells. Nat Commun 2018;9:188.

35. Zhimulev IF, Zykova TY, Goncharov FP et al. Genetic Organization of Interphase Chromosome Bands and Interbands in Drosophila melanogaster. PLOS ONE 2014;9:e101631.

36. Stadler MR, Haines JE, Eisen MB. Convergence of topological domain boundaries, insulators, and polytene interbands revealed by high-resolution mapping of chromatin contacts in the early Drosophila melanogaster embryo. Spradling AC (ed.). eLife 2017;6:e29550.

37. Eagen KP, Hartl TA, Kornberg RD. Stable chromosome condensation revealed by chromosome conformation capture. Cell 2015;163:934-46.

38. Meadows LA, Chan YS, Roote J et al. Neighbourhood Continuity Is Not Required for Correct Testis Gene Expression in Drosophila. PLOS Biol 2010;8:e1000552.

460 39. Lee H, Cho D-Y, Whitworth C et al. Effects of Gene Dose, Chromatin, and Network 461 Topology on Expression in Drosophila melanogaster. PLOS Genet 2016;12:e1006295.

462 40. Ghavi-Helm Y, Jankowski A, Meiers S et al. Highly rearranged chromosomes reveal 463 uncoupling between genome topology and gene expression. Nat Genet 2019:1.

464 41. Ghavi-Helm Y, Klein FA, Pakozdi T et al. Enhancer loops appear stable during development and are associated with paused polymerase. Nature 2014;512:96-100.

42. Maksimenko O, Golovnin A, Georgiev P. Enhancer-Promoter Communication Is Regulated by Insulator Pairing in a Drosophila Model Bigenic Locus. Mol Cell Biol 2008;28:5469-77.

43. Chen H, Levo M, Barinov L et al. Dynamic interplay between enhancer-promoter topology and gene activity. Nat Genet 2018;50:1296-303.

44. Ogiyama Y, Schuettengruber B, Papadopoulos GL et al. Polycomb-Dependent Chromatin Looping Contributes to Gene Silencing during Drosophila Development. Mol Cell 2018;71:7388.e5.

45. Cléard F, Moshkin Y, Karch F et al. Probing long-distance regulatory interactions in the Drosophila melanogaster bithorax complex using Dam identification. Nat Genet 2006;38:931.

475 46. Lanzuolo C, Roure V, Dekker J et al. Polycomb response elements mediate the formation of 476 chromosome higher-order structures in the bithorax complex. Nat Cell Biol 2007;9:1167-74.

477 47. Tolhuis $\mathrm{B}$, Blom M, Kerkhoven RM et al. Interactions among Polycomb Domains Are 478 Guided by Chromosome Architecture. PLOS Genet 2011;7:e1001343. 
481 49. Entrevan M, Schuettengruber B, Cavalli G. Regulation of Genome Architecture and Function 482 by Polycomb Proteins. Trends Cell Biol 2016;26:511-25.

483 50. Luzhin AV, Flyamer IM, Khrameeva EE et al. Quantitative differences in TAD border 484 strength underly the TAD hierarchy in Drosophila chromosomes. $J$ Cell Biochem 485 2019;120:4494-503.

486 51. Hug CB, Grimaldi AG, Kruse K et al. Chromatin Architecture Emerges during Zygotic 487 Genome Activation Independent of Transcription. Cell 2017;169:2 16-228.e19.

488 52. Schubert T, Pusch MC, Diermeier S et al. Df31 protein and snoRNAs maintain accessible 489 higher-order structures of chromatin. Mol Cell 2012;48:434-44.

490 53. Mourad R, Cuvier O. Computational Identification of Genomic Features That Influence 3D 491 Chromatin Domain Formation. PLoS Comput Biol 2016;12, DOI: 10.1371/journal.pcbi.1004908.

492 54. Cubeñas-Potts C, Rowley MJ, Lyu X et al. Different enhancer classes in Drosophila bind 493 distinct architectural proteins and mediate unique chromatin interactions and 3D architecture. 494 Nucleic Acids Res 2017;45:1714-30.

495 55. Li L, Lyu X, Hou C et al. Widespread Rearrangement of 3D Chromatin Organization 496 Underlies Polycomb-Mediated Stress-Induced Silencing. Mol Cell 2015;58:216-31.

497 56. Mateo LJ, Murphy SE, Hafner A et al. Visualizing DNA folding and RNA in embryos at 498 single-cell resolution. Nature 2019;568:49-54.

499 57. Kellum R, Schedl P. A position-effect assay for boundaries of higher order chromosomal 500 domains. Cell 1991;64:941-50.

501 58. Kellum R, Schedl P. A group of scs elements function as domain boundaries in an enhancer502 blocking assay. Mol Cell Biol 1992;12:2424-31.

503 59. Holdridge C, Dorsett D. Repression of hsp70 heat shock gene transcription by the suppressor 504 of hairy-wing protein of Drosophila melanogaster. Mol Cell Biol 1991;11:1894-900.

505 60. Geyer PK, Corces VG. DNA position-specific repression of transcription by a Drosophila 506 zinc finger protein. Genes Dev 1992;6:1865-73.

507 61. Roseman RR, Pirrotta V, Geyer PK. The su(Hw) protein insulates expression of the 508 Drosophila melanogaster white gene from chromosomal position-effects. EMBO J 1993;12:43550942.

510 62. Sigrist CJ, Pirrotta V. Chromatin insulator elements block the silencing of a target gene by the 511 Drosophila polycomb response element (PRE) but allow trans interactions between PREs on 512 different chromosomes. Genetics 1997;147:209-21. 
513 63. Mallin DR, Myung JS, Patton JS et al. Polycomb group repression is blocked by the 514 Drosophila suppressor of Hairy-wing [su(Hw)] insulator. Genetics 1998;148:331-9.

515 64. Recillas-Targa F, Pikaart MJ, Burgess-Beusse B et al. Position-effect protection and enhancer 516 blocking by the chicken beta-globin insulator are separable activities. Proc Natl Acad Sci U S A $517 \quad 2002 ; 99: 6883-8$.

518 65. Kahn TG, Schwartz YB, Dellino GI et al. Polycomb complexes and the propagation of the methylation mark at the Drosophila ubx gene. J Biol Chem 2006;281:29064-75.

66. Van Bortle K, Nichols MH, Li L et al. Insulator function and topological domain border strength scale with architectural protein occupancy. Genome Biol 2014;15:R82.

67. Merkenschlager M, Nora EP. CTCF and Cohesin in Genome Folding and Transcriptional 523 Gene Regulation. Annu Rev Genomics Hum Genet 2016;17:17-43.

524 68. Kyrchanova O, Georgiev P. Chromatin insulators and long-distance interactions in 525 Drosophila. FEBS Lett 2014;588:8-14.

526 69. Nègre N, Brown CD, Shah PK et al. A comprehensive map of insulator elements for the 527 Drosophila genome. PLoS Genet 2010;6:e1000814.

528 70. Schwartz YB, Linder-Basso D, Kharchenko PV et al. Nature and function of insulator protein binding sites in the Drosophila genome. Genome Res 2012;22:2188-98.

530 71. Gambetta MC, Furlong EEM. The Insulator Protein CTCF Is Required for Correct Hox Gene 531 Expression, but Not for Embryonic Development in Drosophila. Genetics 2018;210:129-36.

532 72. Bartkuhn M, Straub T, Herold $\mathrm{M}$ et al. Active promoters and insulators are marked by the 533 centrosomal protein 190. EMBOJ 2009;28:877-88.

534 73. Misulovin Z, Schwartz YB, Li X-Y et al. Association of cohesin and Nipped-B with 535 transcriptionally active regions of the Drosophila melanogaster genome. Chromosoma $536 \quad 2008 ; 117: 89-102$.

537 74. Fay A, Misulovin Z, Li J et al. Cohesin Selectively Binds and Regulates Genes with Paused 538 RNA Polymerase. Curr Biol 2011;21:1624-34.

539 75. Schaaf CA, Kwak H, Koenig A et al. Genome-Wide Control of RNA Polymerase II Activity 540 by Cohesin. PLOS Genet 2013;9:e1003382.

541 76. Rollins RA, Morcillo P, Dorsett D. Nipped-B, a Drosophila Homologue of Chromosomal 542 Adherins, Participates in Activation by Remote Enhancers in the cut and Ultrabithorax Genes. 543 Genetics 1999;152:577-93.

544 77. Schaaf CA, Misulovin Z, Gause M et al. Cohesin and Polycomb Proteins Functionally 545 Interact to Control Transcription at Silenced and Active Genes. PLOS Genet 2013;9:e1003560. 
78. Rowley MJ, Lyu X, Rana V et al. Condensin II Counteracts Cohesin and RNA Polymerase II in the Establishment of 3D Chromatin Organization. Cell Rep 2019;26:2890-2903.e3.

79. Gilbert MK, Tan YY, Hart CM. The Drosophila boundary element-associated factors BEAF32A and BEAF-32B affect chromatin structure. Genetics 2006;173:1365-75.

80. Deng H, Zhang W, Bao X et al. The JIL-1 kinase regulates the structure of Drosophila polytene chromosomes. Chromosoma 2005;114:173-82.

81. Gan M, Moebus S, Eggert $\mathrm{H}$ et al. The Chriz-Z4 complex recruits JIL-1 to polytene chromosomes, a requirement for interband-specific phosphorylation of $\mathrm{H} 3 \mathrm{~S} 10$. J Biosci 2011;36:425-38.

82. Mourad R, Cuvier O. TAD-free analysis of architectural proteins and insulators. Nucleic Acids Res 2018;46:e27-e27.

83. Foe VE, Alberts BM. Studies of nuclear and cytoplasmic behaviour during the five mitotic cycles that precede gastrulation in Drosophila embryogenesis. J Cell Sci 1983;61:31-70.

84. Cardozo Gizzi AM, Cattoni DI, Fiche J-B et al. Microscopy-Based Chromosome Conformation Capture Enables Simultaneous Visualization of Genome Organization and Transcription in Intact Organisms. Mol Cell 2019;74:212-222.e5.

85. McDaniel SL, Gibson TJ, Schulz KN et al. Continued Activity of the Pioneer Factor Zelda Is Required to Drive Zygotic Genome Activation. Mol Cell 2019;74:185-195.e4.

86. Schulz KN, Bondra ER, Moshe A et al. Zelda is differentially required for chromatin accessibility, transcription factor binding, and gene expression in the early Drosophila embryo. Genome Res 2015;25:1715-26.

87. Chathoth KT, Zabet NR. Chromatin architecture reorganization during neuronal cell differentiation in Drosophila genome. Genome Res 2019;29:613-25.

88. Heist T, Fukaya T, Levine M. Large distances separate coregulated genes in living Drosophila embryos. Proc Natl Acad Sci 2019:201908962.

89. Boettiger AN, Bintu B, Moffitt JR et al. Super-resolution imaging reveals distinct chromatin folding for different epigenetic states. Nature 2016;529:418-22.

90. Szabo Q, Jost D, Chang J-M et al. TADs are 3D structural units of higher-order chromosome organization in Drosophila. Sci Adv 2018;4:eaar8082.

91. Jost D, Carrivain P, Cavalli G et al. Modeling epigenome folding: formation and dynamics of topologically associated chromatin domains. Nucleic Acids Res 2014;42:9553-61.

92. Lesage A, Dahirel V, Victor J-M et al. Polymer coil-globule phase transition is a universal folding principle of Drosophila epigenetic domains. Epigenetics Chromatin 2019;12:28. 
579 93. Ea V, Sexton $\mathrm{T}$, Gostan $\mathrm{T}$ et al. Distinct polymer physics principles govern chromatin 580 dynamics in mouse and Drosophila topological domains. BMC Genomics 2015;16:607.

581 94. Shimamoto $\mathrm{Y}$, Tamura $\mathrm{S}$, Masumoto $\mathrm{H}$ et al. Nucleosome-nucleosome interactions via 582 histone tails and linker DNA regulate nuclear rigidity. Mol Biol Cell 2017;28:1580-9.

583 95. Michieletto D, Orlandini E, Marenduzzo D. Polymer model with Epigenetic Recoloring 584 Reveals a Pathway for the de novo Establishment and 3D Organization of Chromatin Domains. 585 Phys Rev X 2016;6:041047.

586 96. Ghosh SK, Jost D. How epigenome drives chromatin folding and dynamics, insights from 587 efficient coarse-grained models of chromosomes. PLoS Comput Biol 2018;14, DOI: $588 \quad 10.1371$ journal.pcbi.1006159.

589 97. Jost D, Vaillant C. Epigenomics in 3D: importance of long-range spreading and specific 590 interactions in epigenomic maintenance. Nucleic Acids Res 2018;46:2252-64. 


\section{Figure legends}

593 Figure 1: Hierarchical organisation of the Drosophila genome.

594 a. Chromosomes are compacted with in the nuclei in a Rabl-like configuration, i.e. the centromeres of the 595 chromosomes cluster together to one pole of the nucleus in close proximity to the nucleolus, while the 596 telomeres are found in the opposite pole. b. Chromosomes are divided in small and compact domains 597 called topologically-associated domains (or TADs) that cluster together according to their chromatin 598 state and form the $\mathrm{A}$ (blue) and the B (pink) compartments. c. TADs bring into close proximity genes and 599 their cis-regulatory regions and are thought to form isolated transcriptional units within which the 600 expression of genes is correlated. TADs are delimited by the presence of architectural protein binding 601 sites at their borders. Drosophila TADs form relatively few loop domains compared to mammals, thus 602 contacts between borders might be less frequent. d. Within TADs, enhancers are brought into close 603 proximity to the promoter of the genes they regulate through loops that can be mediated by insulator 604 pairing or RNA Pol II pausing. Active enhancers and promoters are bound by RNA Pol II, transcription 605 factors (TFs) and co-factors.

606 Figure 2: Schematic representation of Hi-C contact maps of inactive and active Drosophila genome 607 states.

608 Before zygotic genome activation (ZGA) and during cell cycle, when the genome is transcriptionally 609 inactive, the Drosophila genome is unstructured, as shown by uniform contact frequencies in $\mathrm{Hi}-\mathrm{C}$ 610 contact maps. At the onset of ZGA, weak TADs, loops, and compartments start to appear and gradually 611 become stronger at later stages of development. TAD formation is concomitant with the transcription of 612 the genes present at their borders. 


\section{Prior to ZGA and during mitosis}

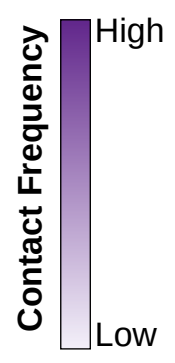

\section{During ZGA}

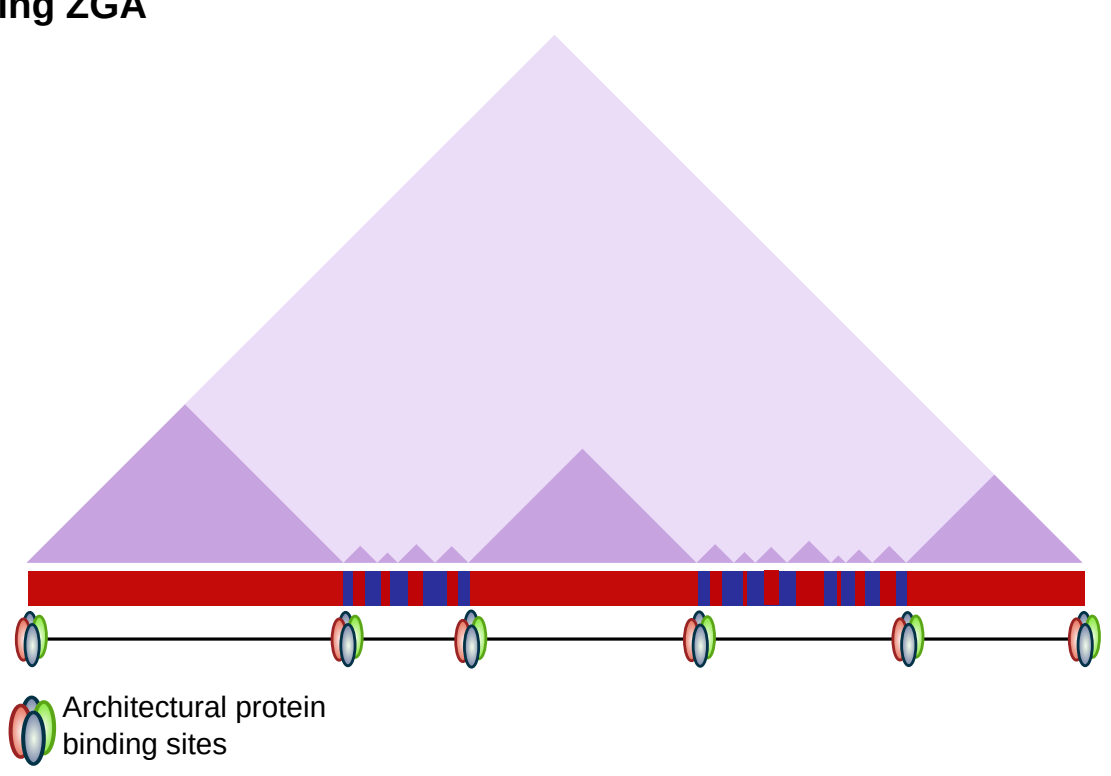

After ZGA and in differentiated cells

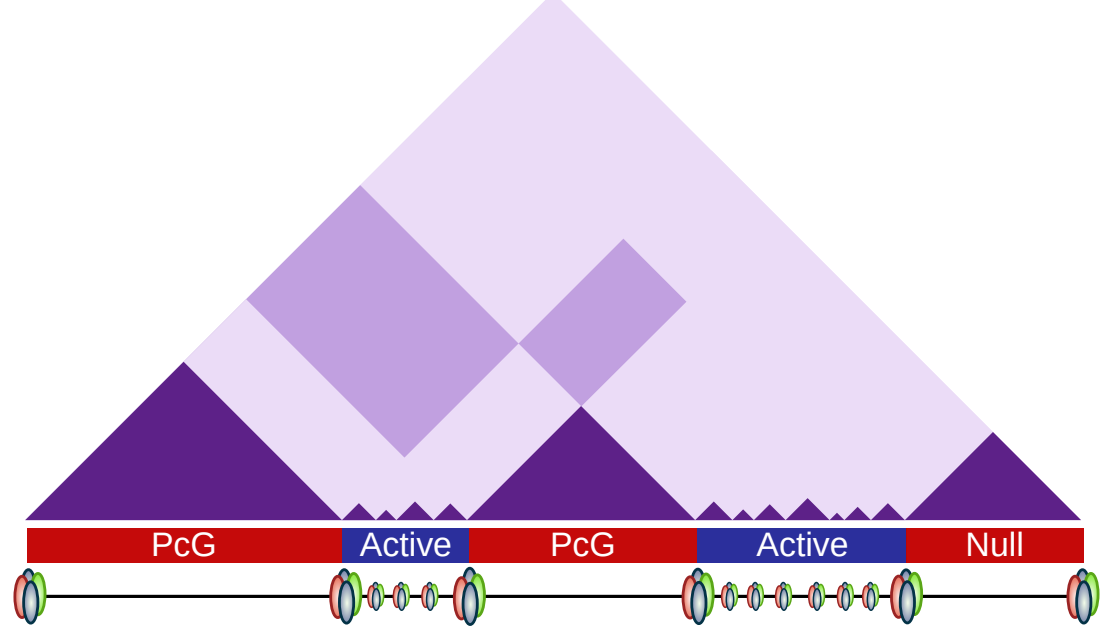

